\title{
Transcatheter aortic valve implantation: from revolution to evolution
}

\author{
Edgar Lik Wui Tay ${ }^{1,2}$, MBBS, MRCP, Kian-Keong $\underline{\operatorname{Poh}}^{1,2}$, FRCP, FACC, James Wei Luen $\underline{\text { Yip }}^{1,2}$, MBBS, MRCP, \\ William KF Kong $^{1}$, MBChB, FRACP
}

$\mathrm{T}$ he advent of transcatheter aortic valve implantation has transformed the treatment of aortic valve stenosis. This disease, which previously could only be managed by openheart surgery, can now be treated via a percutaneous technique.

The terms transcatheter aortic valve implantation (TAVI), first coined by the Europeans and Canadians, and transcatheter aortic valve replacement, preferred by American cardiologists, are used interchangeably. The procedure consists of the delivery of an aortic valve prosthesis crimped into a smaller profile (very similarly to how coronary stents are crimped) through the femoral artery, or occasionally through the apex of the left ventricle or subclavian artery; it is subsequently positioned and deployed at the site of the diseased aortic valve. The stent frame's radial strength effectively displaces the native valve leaflets and the new valve leaflets sutured within the stent frame then begin to function.

Initial pivotal trials tested this new technology in surgically inoperable patients, followed by high-risk surgical patients. The results were stunning. There was a significant reduction in mortality of the inoperable cohort. At the one-year follow-up, there was a $20 \%$ absolute reduction in mortality and an $18.3 \%$ reduction in the combined endpoint of death or stroke in patients who underwent TAVI. In TAVI patients, the number needed to treat in order to prevent a death was 5.0 and that to prevent a death or a major stroke was 5.5. In the high-risk cohort, TAVI was not found to be inferior to surgery..$^{(1,2)}$ This led to changes in the guidelines of the American College of Cardiology and European Society of Cardiology, which both recommended TAVI for inoperable and high-risk patients. ${ }^{(3,4)}$

Initial concerns regarding the new technology included the possibility of increased paravalvular leak, increased need for pacemaker implantation, and higher likelihood of stroke and major vascular complications due to the large size of the catheters required for the procedure. These challenges were addressed through significant successes over the past few years. Possible reasons for these successes include: the formation of a heart team that reviewed and treated patients who were most likely to benefit from TAVI; numerous publications and sharing of TAVI techniques; concomitant improvement of the procedure with reduction in sheath sizes; improving the seal around the annulus to prevent paravalvular leak; and a better understanding of the importance of implant depth as well as the retrievability of modern TAVI valves. The imaging techniques of echocardiography and, later, computed tomography to understand the patient's root anatomy also contributed to the success of TAVI.

A decade on, TAVI has matured as a procedure. The revolution may have reached a plateau now that the technology has stabilised and safety has markedly improved. However, its next phase of evolution will be exciting. This treatment has recently been extended to individuals at lower risk (i.e. patients with intermediate surgical risk). A few months ago, a randomised clinical trial confirmed that TAVI was noninferior to surgery in this cohort. ${ }^{(5)}$ Another recently published study showed that the latest third-generation TAVI device may be superior to open surgery. ${ }^{(6)}$ This led to the United States Food and Drug Administration's approval of a low-risk clinical trial. If the results of this trial are positive, it is plausible that TAVI could become a viable alternative treatment for most patients.

TAVI is also currently being utilised in other groups of patients who were excluded in the initial clinical trials, those with bicuspid aortic valve stenosis or degenerated bioprosthesis. Bicuspid aortic valve stenosis usually occurs about a decade earlier compared to degenerative calcific aortic valve stenosis. Bicuspid valves are more complex in their root anatomy. A multinational collaboration has recently been set up to collect information on the treatment of these patients and details will be published soon.

Since the initial description of a series of successful implantations of transcatheter heart valves in degenerated valves, ${ }^{(7)}$ there has been a significant increase in the adoption of a TAVI-like technique, known as valve-in-valve TAVI. Commendably, Chiam et $\mathrm{al}^{(8)}$ described a case series in an Asian cohort that showed good short-term outcomes; their results were in line with those from the global valve-in-valve registry ${ }^{(9)}$ Like much good research, the study generates more questions than answers. For instance, the number of patients with smaller degenerated bioprostheses is likely to be higher in an Asian cohort. There is a paucity of published Asian data on this subject. The likelihood of patient-prosthesis mismatch is also likely to be higher, with three out of eight patients having severe patient-prosthesis mismatch in this study. Importantly, according to the global registry, a smaller valve prosthesis is a predictor of poorer survival. It is thus imperative that patient selection should be done cautiously by an experienced heart team. Similarly, most other published studies were performed with first-generation balloon expandable or self-expanding devices. Procedural safety and better outcomes are expected with the use of the new-generation devices.

${ }^{1}$ Department of Cardiology, National University Heart Centre, National University Health System, ${ }^{2}$ Department of Medicine, Yong Loo Lin School of Medicine, National University of Singapore, Singapore

Correspondence: Dr Edgar Tay, Senior Consultant Cardiologist, Department of Cardiology, National University Heart Centre, National University Health System, 1 E Kent Ridge Road, NUHS Tower Block, Level 9, Singapore 119228. edgar_tay@nuhs.edu.sg 
That said, what impact does the feasibility of valve-in-valve TAVI have on heart valve disease patients? Firstly, it provides a treatment option for some of the extreme high-risk individuals undergoing redo surgery who would previously have been relegated to palliative care. Appropriate patients have shown demonstrable improvement in New York Heart Association classification status following valve-in-valve TAVI. Secondly, more thought may be given to selection of the prosthetic valve during the initial surgical valve surgery. Surgeons may now have to consider the future possibility of valve-in-valve TAVI in such patients. It is plausible, therefore, that younger patients may be offered surgical bioprostheses with a view towards 'extending the lifespan' of the heart valve through valve-in-valve TAVI should it degenerate over time, as opposed to choosing a metallic heart valve and requiring anticoagulation. ${ }^{(10)}$ In addition, surgeons may also choose larger valves or those that are more compatible with future valve-in-valve TAVI.

With the rapid increase in TAVI procedures in Asia in the past two years, it is now the right time for Asian countries to collaborate on longer-term prospective research on both TAVI and valve-in-valve TAVI, to determine their impact on Asian patients.

\section{REFERENCES}

1. Leon MB, Smith CR, Mack M, et al; PARTNER Trial Investigators. Transcatheter aortic-valve implantation for aortic stenosis in patients who cannot undergo surgery. N Engl J Med 2010; 363:1597-607.

2. Smith CR, Leon MB, Mack MJ, et al; PARTNER Trial Investigators. Transcatheter versus surgical aortic-valve replacement in high-risk patients. N Engl J Med 2011; 364:2187-98.

3. Joint Task Force on the Management of Valvular Heart Disease of the European Society of Cardiology (ESC), European Association for CardioThoracic Surgery (EACTS); Vahanian A, Alfieri O, Andreotti F, et al. Guidelines on the management of valvular heart disease (version 2012). Eur Heart J 2012; 33:2451-96.

4. Nishimura RA, Otto CM, Bonow RO, et al; American College of Cardiology/American Heart Association Task Force on Practice Guidelines. 2014 AHA/ACC guideline for the management of patients with valvular heart disease: a report of the American College of Cardiology/ American Heart Association Task Force on Practice Guidelines. J Am Coll Cardiol 2014; 63:e57-185.

5. Leon MB, Smith CR, Mack MJ, et al; PARTNER 2 Investigators. Transcatheter or Surgical Aortic-Valve Replacement in Intermediate-Risk Patients. N Engl J Med 2016; 374:1609-20.

6. Thourani VH, Kodali S, Makkar RR, et al. Transcatheter aortic valve replacement versus surgical valve replacement in intermediate-risk patients: a propensity score analysis. Lancet 2016; 387:2218-25.

7. Gurvitch R, Cheung A, Ye J, et al. Transcatheter valve-in-valve implantation for failed surgical bioprosthetic valves. J Am Coll Cardiol 2011; 58:2196-209.

8. Chiam PT, Ewe SH, Soon JL, et al. Percutaneous transcatheter aortic valve implantation for degenerated surgical bioprostheses: the first case series in Asia with one-year follow-up. Singapore Med J 2016; 57:401-5.

9. Dvir D, Webb JG, Bleiziffer S, et al. Valve-in-Valve International Data Registry Investigators. Transcatheter aortic valve implantation in failed bioprosthetic surgical valves. JAMA 2014; 312:162-70.

10. Azadani AN, Tseng EE. Transcatheter heart valves for failing bioprostheses: state-of-the-art review of valve-in-valve implantation. Circ Cardiovasc Interv 2011; 4:621-8. 